\title{
Die Confessio Augustana in den offiziellen ökumenischen Dialogen
}

Im Folgenden soll ein Überblick gegeben werden, wie und in welchen Kontexten die Confessio Augustana (CA) in den offiziellen ökumenischen Dialogen auftaucht bzw. behandelt wird und welche Aspekte dabei zur Sprache kommen. Dabei konzentriere ich mich auf die internationalen Dialoge, auch wenn selbstverständlich eine Ausweitung auf die nationalen Dialoge, beispielsweise die beiden ersten Dokumente der Bilateralen Arbeitsgruppe der Deutschen Bischofskonferenz und der Kirchenleitung der Vereinigten Evangelisch-Lutherischen Kirche Deutschlands oder den lutherisch-katholischen Dialog in den USA und in Finnland in einem weiteren Schritt durchaus sinnvoll wäre. Konkret werden die Dialoge des Lutherischen Weltbundes (LWB) mit anderen Kirchen oder Kirchenbünden daraufhin untersucht, welche Beobachtungen sich machen lassen, wenn man der Frage nachgeht, wie das Augsburger Bekenntnis verwendet wird, in welchen Kontexten es zur Sprache kommt und ob und in welcher Weise auf die Rolle dieses Bekenntnisses reflektiert wird.

\section{Die Confessio Augustana als verbindliches Bekenntnis der lutherischen Kirchen}

Der erste und sicherlich selbstverständliche Punkt ist, dass die Confessio Augustana immer wieder neben den drei altkirchlichen Bekenntnissen, die am Beginn des Konkordienbuches stehen, und dem Kleinen Katechismus als verbindliche Bekenntnisse der lutherischen Kirchen und damit, wie es im Dialog mit den Mennoniten an einer Stelle heißt, auch als „Band [...], das die lutherischen Kirchen im Lutherischen Weltbund miteinander verbindet“, ${ }^{1}$ benannt wird. So heißt es etwa im Dialog mit den Adventisten:

Die Kirchen der lutherischen Reformation halten an fünf Bekenntnissen bzw. Bekenntnisschriften fest: Den altkirchlichen Bekenntnissen (Apostolikum, Nicaenum, Athanasium [sic!]), dem Augsburgischen Bekenntnis und Luthers Kleinem Katechismus. Das apostolische Bekenntnis und das Bekenntnis von Nicaea haben einen selbstverständlichen Platz in der

1 Heilung der Erinnerungen - Versöhnung in Christus. Bericht der Internationalen lutherischmennonitischen Studienkommission, 2005-2008, in: DwÜ 4, 401-506, Nr. 2 (407).

Ә OpenAccess. (c) 2022 Burkhard Neumann, publiziert von De Gruyter. (cc))BY-NC-ND Dieses Werk ist lizenziert unter einer Creative Commons Namensnennung - Nicht kommerziell - Keine Bearbeitung 4.0 International Lizenz. https://doi.org/10.1515/9783110683868-021 
Liturgie der lutherischen Kirchen. Luthers Kleiner Katechismus wird in der allgemeinen christlichen Unterweisung benutzt, und das Augsburgische Bekenntnis ist vorrangig für die theologische Ausbildung und Orientierung von Bedeutung. ${ }^{2}$

Dass hier nur die CA, und zwar die Invariata, und Luthers Kleiner Katechismus genannt werden bzw. dass an anderer Stelle gesagt werden kann, dass ihnen im Rahmen des Konkordienbuches „eine zentrale Rolle“3 ${ }^{\text {“3 }}$ kommt, liegt daran, dass laut der Lehrgrundlage des Lutherischen Weltbundes diese beiden Bekenntnisse besonders hervorgehoben werden, weil sie in allen Kirchen des LWB anerkannt werden:

Der Lutherische Weltbund bekennt die Heilige Schrift des Alten und Neuen Testamentes als die alleinige Quelle und Norm seiner Lehre, seines Lebens und seines Dienstes. Er sieht in den drei ökumenischen Glaubensbekenntnissen und in den Bekenntnissen der lutherischen Kirche, insbesondere in der unveränderten Augsburgischen Konfession und in dem Kleinen Katechismus Martin Luthers eine zutreffende Auslegung des Wortes Gottes. ${ }^{4}$

Selbstverständlich wird dabei, wie eben auch in der Verfassung des Lutherischen Weltbundes, immer herausgestellt, dass diese Bekenntnisse unter der Heiligen Schrift als der norma normans stehen, sie „eine unter der Schriftnorm liegende Norm“ ${ }^{\text {5 }}$ darstellen, aber in dieser Funktion eine „getreue Darlegung der Lehre der Heiligen Schrift und der Alten Kirche"6 bilden. Auf den letzten Punkt, den Bezug zur Alten Kirche, werde ich am Ende noch einmal zu sprechen kommen.

2 Adventisten und Lutheraner im Gespräch. Bericht über die Gespräche zwischen dem Lutherischen Weltbund und der Kirche der Siebenten-Tags-Adventisten, 1994-1998, in: DwÜ 3, 77-109, Nr. 43 (86); vgl. Communion: On Being the Church. Report of the Lutheran-Reformed Joint Commission between the Lutheran World Federation (LWF) and the World Communion of Reformed Churches (WCRC), 2006-2012, Genf 2014, Nr. 146 (42), wo nach den Nennung dieser beiden Bekenntnisse alle Schriften des Konkordienbuches als „integraler Teil“ ihrer Tradition aufgeführt werden.

3 Wachsende Gemeinschaft. Bericht der Internationalen anglikanisch-lutherischen Arbeitsgruppe, 2000 -2002, in: DwÜ 4, 129-193, Nr. 100 (154).

4 Artikel II der Verfassung des LWB: https://de.lutheranworld.org/sites/default/files/documents/ Constitution\%20DE\%20final.pdf (16.06.2021).

5 Die Apostolizität der Kirche. Studiendokument der Lutherisch/Römisch-katholischen Kommission für die Einheit, in: DwÜ 4, 527-678, Nr. 374 (650).

6 Die Kirche: Gemeinschaft der Gnade. Bericht der Gemeinsamen Lutherisch/Methodistischen Kommission, 1984, in: DwÜ 2, 231-257, Nr. 19 (236). 


\section{Die Aussagen der Confessio Augustana als Kriterium der Kirchlichkeit}

Darüber hinaus wird das Augsburger Bekenntnis im ökumenischen Dialog auch ausdrücklich als Maßstab oder Kriterium der Kirchlichkeit verstanden. Diese Rolle der lutherischen Bekenntnisse und vor allem der CA kommt im Wesentlichen in zwei Kontexten vor:

a) Zum einen findet sie sich dort, wo es gilt, zunächst die Grundlagen eines gemeinsamen Dialogs und einer gemeinsamen christlichen Basis festzustellen, wo es also, anders gesagt, darum geht, ob man gemäß Artikel 7 der CA im Verständnis des Evangeliums übereinstimmt. Dies wird zwar faktisch in meisten Dialogen bereits vorausgesetzt und im Verlauf eines solchen Dialogs dann entsprechend festgehalten. ${ }^{7}$ Aber, und das zeigt sich am Gespräch zwischen Adventisten und Lutheranern, es kann durchaus der Fall sein, dass der Dialog diesen Sachverhalt, d.h. die grundsätzliche Übereinstimmung im Evangelium, zunächst einmal ausdrücklich klären muss. ${ }^{8}$ Darum wird hier nicht nur allgemein auf die Rolle der drei altkirchlichen Bekenntnisse, der Confessio Augustana und Luthers Kleinem Katechismus als den grundlegenden und verbindlichen Bekenntnissen der lutherischen Kirche hingewiesen, sondern es wird ausdrücklich auf der Grundlage von CA 7 als einem für die Lutheraner maßgeblichen Kriterium eine „Übereinstimmung in der zentralen christlichen Botschaft von der Rechtfertigung allein durch den Glauben"9 festgestellt, die, verbunden mit der Frage nach der Taufe, auf die ich in einem anderen Kontext noch zu sprechen kommen werde, und dem Ausschluss eines exklusivistischen Kirchenverständnissen auf adventistischer Seite zu der abschließenden Empfehlung führt,

dass die Lutheraner in dem jeweiligen nationalen und regionalen Umfeld ihrer Kirchen die Siebenten-Tags-Adventisten nicht als Sekte, sondern als Freikirche und weltweite, christliche Gemeinschaft behandeln. Diese Empfehlung gründet sich zum einen auf das adventistische Verständnis der Wassertaufe im Namen des Dreieinigen Gottes, einem Verständnis, das für Lutheraner bedeutet, dass diese Taufe gültig ist, wie auch ferner auf der gemeinsamen Überzeugung, dass ,wahre Christen auch in anderen Kirchen gefunden werden können“ [...], einer Sicht, die mit CA VIII vereinbar ist. ${ }^{10}$

7 Vgl. etwa: Communion: On Being the Church, Nr. 106-113 (35f.)

8 Vgl. Adventisten und Lutheraner im Gespräch.

9 Adventisten und Lutheraner im Gespräch, Nr. 22 (82).

10 Adventisten und Lutheraner im Gespräch, Nr. 94 (94). 
Die Confessio Augustana stellt hier also die Grundlage dar, um unbeschadet weiterhin bestehender Differenzen von der grundsätzlichen Christlichkeit adventistischer Lehre auszugehen und damit auch den kirchlichen Charakter der Siebten-Tags-Adventisten anzuerkennen.

In diesem Zusammenhang ist es m. E. bezeichnend, dass diese Funktion des Augsburger Bekenntnisses im internationalen lutherisch/katholischen Dialog nie angesprochen bzw. problematisiert worden ist. Vielmehr ist man auch hier faktisch immer schon von einer solchen grundlegenden Übereinstimmung im Evangelium und d.h. nach lutherischem Verständnis auch in der Rechtfertigungslehre ausgegangen. Bereits im so genannten Malta-Bericht Das Evangelium und die Kirche wird ja nicht nur eine solche grundlegende Übereinstimmung in der Rechtfertigungslehre angesprochen, ${ }^{11}$ die dann nach langen Vorarbeiten 1999 in der Gemeinsamen Erklärung zur Rechtfertigungslehre verbindlich festgehalten werden konnte, ${ }^{12}$ sondern es wird dort auch bereits ausdrücklich festgestellt, dass die lutherischen Bekenntnisschriften „den kirchlichen Charakter der römischkatholischen Gemeinschaft “13 betonen, d.h. dass sie also das voraussetzen, was sie mit manchen anderen Kirchen, wie etwa den Adventisten, erst klären müssen.

b) Der zweite Sachverhalt, in dem diese kriterielle Funktion der CA ausdrücklich behandelt wird, ist die Frage nach konkreten Schritten auf dem Weg zur Kirchengemeinschaft. Hier geht es darum, die verschiedenen Bekenntnisse bzw. Bekenntnistraditionen miteinander in Einklang zu bringen bzw. danach zu fragen, inwieweit und auf welcher Grundlage eine Kirchengemeinschaft bekenntnisverschiedener Kirchen möglich ist und wie sie auszusehen habe. Auf internationaler Ebene wird das vor allem im Dialog zwischen Lutheranern und Anglikanern sowie zwischen Lutheranern und Reformierten behandelt, und zwar auch und gerade deshalb, weil es auf regionaler Ebene bereits zahlreiche Formen von Kirchengemeinschaft dieser Kirchen untereinander gibt, die sich aber aus unterschiedlichen Gründen auf Weltebene noch nicht verwirklichen lassen. ${ }^{14}$ In beiden Dialogen sieht man auf dieser Ebene in den unterschiedlichen Bekennt-

11 Bericht der Evangelisch-lutherisch/Römisch-katholischen Studienkommission, Das Evangelium und die Kirche, 1972 („Malta-Bericht“), in: DwÜ 1, 248-271, Nr. 26 (255).

12 Gemeinsame Erklärung zur Rechtfertigungslehre des Lutherischen Weltbundes und der Katholischen Kirche, in: DwÜ 3, 419-441. Zwar stimmt es, was in der Fußnote zu Nr. 1 gesagt wird, dass die CA und Luthers Kleiner Katechismus, die von einigen lutherischen Kirchen als alleinige verbindliche Lehrgrundlage anerkannt werden, „keine die Rechtfertigungslehre betreffenden Lehrverurteilungen gegenüber der Römisch-Katholischen Kirche“ (419) enthalten, aber die Frage nach der Übereinstimmung im Evangelium beschränkt sich ja nicht allein auf die Aufarbeitung gegenseitiger Lehrverurteilungen, gerade wenn man vom gegenwärtigen Stand der Lehre ausgeht. 13 Das Evangelium und die Kirche, Nr. 64 (265)

14 Vgl. Wachsende Gemeinschaft; Communion: On Being the Church. 
nissen und ihrer jeweiligen Rolle in den beiden Kirchen keine kirchentrennende Differenz mehr, sondern einen Ausdruck einer versöhnten und damit legitimen Vielfalt. ${ }^{15}$ Während aber der lutherisch-reformierte Dialog ausgehend von der Einheit im Verständnis des Evangeliums davon spricht, dass darum beide Kirchen in Gemeinschaft miteinander stehen (,we are in communion“) ${ }^{16}$ und entsprechende praktische Schritte empfiehlt, um diese Gemeinschaft besser zum Ausdruck zu bringen, wird im Gespräch zwischen Lutheranern und Anglikanern ausdrücklich darauf hingewiesen, dass es neben diesen Punkten noch ein Reihe von anderen Fragen gibt, die auf dieser internationalen Ebene noch keine volle Kirchengemeinschaft möglich machen. ${ }^{17}$

\section{Die Confessio Augustana im Kontext einer dezidierten Bekenntnishermeneutik}

Ein weiterer Aspekt, in dem das Augsburger Bekenntnis in den internationalen Dialogen behandelt wird, ist die Frage nach einer sachgerechten historischen und theologischen Auslegung einzelner Artikel, also die Frage nach einer sachgerechten Bekenntnishermeneutik.

Der konkrete Anlass für eine entsprechende Untersuchung sind im Rahmen des internationalen ökumenischen Dialogs die Verwerfungen der „Wiedertäufer“ in der Confessio Augustana, die im Dialog mit jenen Kirchen, die sich in der Tradition der „Wiedertäufer“ sehen, problematisiert werden. Während im lutherisch/katholischen Dialog über Kirche und Rechtfertigung die Verurteilungen der

15 Vgl. Wachsende Gemeinschaft, Nr. 138: „Eine ausreichende Übereinstimmung im Glauben verpflichtet uns nicht, uns, jede lehrmäßige Formulierung zu eigen zu machen, die für die jeweils andere unserer verschiedenen Traditionen charakteristisch ist" (Porvoo § 33).“ (166); Nr. 150: „Unsere Arbeitsgruppe hat nicht den Versuch unternommen, alle Fragen aufzulisten, die sich gestellt haben oder die sich im Rahmen des anglikanisch-lutherischen Dialogs noch stellen werden. Es gab jedoch drei Themenschwerpunkte, die die beiden Gemeinschaften ihr zur Prüfung unterbreitet hatten: a) der Stellenwert der grundlegenden Bekenntnistexte; b) die Formulierung des Ziels der Einheit; c) die historische Bischofssukzession als Zeichen der Apostolizität der Kirche. In einem früheren Kapitel des vorliegenden Berichts ist die Arbeitsgruppe zu dem Schluss gelangt, dass die Anomalien, die in diesen Bereichen beim Ausdruck und der Formulierung von Übereinstimmungen bestehen, als hinnehmbar empfunden werden, ja dass sich ein klarer Konsens abzeichnet.“ (169); vgl. ähnlich: Communion: On Being the Church, Nr.149-151 (43f.), wo als Fazit festgehalten wird: „The above mentioned Lutheran and Reformed confessional writings today exist in dogmatically reconciled, hence legitimate, diversity.“ (Nr. 151 [44]).

16 Communion: On Being the Church, Nr. 155 (45).

17 Vgl. Wachsende Gemeinschaft, Nr. 153 (170). 
Wiedertäufer in CA 5 nur genannt und inhaltlich bejaht werden, ohne das eine weiter Reflexion über den historischen Kontext oder die Wirkungsgeschichte dieser Verurteilungen stattfindet, ${ }^{18}$ stellt sich die Frage im Dialog mit den Baptisten und vor allem im Dialog mit den Mennoniten verständlicherweise auf eine ganz andere Weise. Das im Laufe der Zeit gewachsene Problembewusstsein zeigt sich allein schon dadurch, dass der baptistisch/lutherische Dialog diese Frage noch auf etwas mehr als drei Seiten behandeln kann, ${ }^{19}$ während der mennonitisch/lutherische Dialog dieses Problem dann in einer viel tiefer gehenden Gründlichkeit auf insgesamt über 100 Seiten angeht. ${ }^{20}$

Im baptistisch/lutherischen Dialog werden zunächst die Verurteilungen der Täufer in der Confessio Augustana und in der Konkordienformel genannt. ${ }^{21}$ Auf der einen Seite wird festgehalten, dass diese Verwerfungen sich „gegen Lehren und Lehrer [richteten], die dem lutherischen Verständnis des Evangeliums entgegenstanden. “22 Zugleich aber wird anerkannt, dass sie Folgen hatten, die weit über diesen Inhalt hinausgingen und die auch, wie es relativ zurückhaltend gesagt wird, dazu führten, dass die moderne baptistische Bewegung „ihrerseits auch unter Diskriminierung und rechtlichen Schwierigkeiten als Folge der lutherischen Verwerfungen gelitten“23 hat.

Im Blick auf die Geltung der Bekenntnisse wird auf der einen Seite von lutherische Seite die Kontinuität im Glauben festgehalten, andererseits aber un-

18 Vgl. Gemeinsame Römisch-katholische/Evangelisch-lutherische Kommission, Kirche und Rechtfertigung. Das Verständnis der Kirche im Licht der Rechtfertigungslehre, in: DwÜ 3, 317-419, Nr. 125, wo hervorgehoben wird, dass auch die Verkündigung des Evangeliums für Lutheraner „,sakramentalen“ Charakter hat, insofern dem hörbaren Wort die Kraft innewohnt, den Glaubenden jene Wirklichkeit des Heils zu vermitteln, auf die die Worte der Verkündigung hinweisen. [...] Deshalb werden die Wiedertäufer ausdrücklich zurückgewiesen, da sie ,lehren, dass wir den Heiligen Geist ohne das leibhafte Wort des Evangeliums [...] erlangen'. In dieselbe Richtung weist die Ablehnung der Donatisten, nach deren Auffassung die Sakramente durch den Dienst schlechter Spender unnütz und unwirksam werden. Wort und Sakrament bleiben wegen der Einsetzung und des Auftrags Christi wirksam, auch wenn sie von schlechten Spendern mit- und ausgeteilt werden.“ (362) Die entsprechenden Anmerkungen verwiesen auf CA 5 und CA 8.

19 Baptisten und Lutheraner im Gespräch. Eine Botschaft an unsere Kirchen/Gemeinden. Bericht der Gemeinsamen Kommission des Baptistischen Weltbundes und des Lutherischen Weltbundes, 1990, in: DwÜ 2, 189-216, hier 212-215 (Nr. 93-107). Auf dieses Dokument wird auch im adventistisch/lutherschen Dialog hingewiesen, wo es um die Bewertung der Glaubenstaufe geht, vgl. Adventisten und Lutheraner im Gespräch, Nr. 60 (88).

20 Heilung der Erinnerungen - Versöhnung in Christus.

21 Es handelt sich um die Artikel 5, 9, 12, 16 und 17 der CA und Kapitel 12,3 der Epitome der Konkordienformel, vgl. Baptisten und Lutheraner im Gespräch, Nr. 94f. (212f.)

22 Baptisten und Lutheraner im Gespräch, Nr. 97 (213).

23 Baptisten und Lutheraner im Gespräch, Nr. 98 (214). 
terschieden zwischen dem „Geist der Bekenntnisse“24 und jenen menschlichen Formulierungen, die im Konflikt mit dem heutigen Verständnis des Evangeliums stehen. Faktisch bedeutet das, dass inhaltlich nur noch Artikel 9 der CA, d.h. die Frage der Kindertaufe, relevant ist und die theologische Diskussion über ihn weiterhin notwendig bleibt, während die anderen Verurteilungen heute nicht mehr gelten und sie weithin auch schon im 16. Jahrhundert nicht auf die Täufer zutrafen. Damit wird auch immer noch vorkommenden aktuellen Diskriminierungen eine Absage erteilt.

$\mathrm{Zu}$ den abschließenden Empfehlungen ${ }^{25}$ gehört darum der Vorschlag, dass zukünftige Auflagen der Bekenntnisschriften eine Erklärung enthalten sollten, dass die damaligen Verwerfungen heute nicht mehr zutreffen und weiterhin bestehende Unterschiede „mit einem freundlichen Nein behandelt und zum Anlass vertiefter gemeinsamer Studien gemacht werden“ ${ }^{\text {26 }}$ sollen. ${ }^{27}$ Vor allem wird abschließend deutlich unterschieden zwischen einer legitimen Ablehnung einer Lehre und eine illegitimen Verurteilung einer Person.

Diese knappen Ausführungen werden, wie schon gesagt, im lutherisch/mennonitischen Dialog wesentlich breiter und differenzierter behandelt. Ausgangspunkt war das Gedenken der Confessio Augustana 1980, zu der auch die Mennoniten eingeladen wurden und durch das den lutherischen Kirchen bewusst wurde, welche Rolle die Verwerfungen der so genannten „Wiedertäufer“ in der CA sowohl historisch wie theologisch gehabt hatten und z.T. noch heute haben. ${ }^{28}$ Die verschiedenen Dialoge zunächst auf nationaler und dann auf internationaler Ebene führten zu dem genannten Dialogdokument und schließlich zu den beeindruckenden Versöhnungsfeierlichkeiten zwischen Lutheranern und Mennoniten während der 11. Vollversammlung des Lutherischen Weltbundes in Stuttgart $2010 .^{29}$

24 Baptisten und Lutheraner im Gespräch, Nr. 99 (214).

25 Vgl. Baptisten und Lutheraner im Gespräch, Nr. 107 (215).

26 Baptisten und Lutheraner im Gespräch, Nr. 107 (215).

27 Das geschieht in der deutschen Übersetzung: Unser Glaube. Die Bekenntnisschriften der evangelisch-lutherischen Kirche. Ausgabe für die Gemeinde, im Auftrag der Kirchenleitung der Vereinigten Evangelisch-Lutherischen Kirche Deutschlands (VELKD), hg.v. Amt der VELKD, redaktionell betreut v. Johannes Hund u. Hans-Otto Schneider, Gütersloh ${ }^{6} 2013$, 49; 51f. wesentlich deutlicher als in der wissenschaftlichen Neuausgabe der Bekenntnisschriften: Die Bekenntnisschriften der Evangelisch-Lutherischen Kirche. Vollständige Neuedition, hg.v. Irene Dingel im Auftrag der Evangelischen Kirche in Deutschland, Göttingen 2014, 100, Anm. 52.

28 Vgl. Heilung der Erinnerungen - Versöhnung in Christus, Nr. $1 \mathrm{ff}$.

29 Vgl. dazu Rainer W. Burkart/Oliver Schuegraf, Heilung der Erinnerungen - Versöhnung in Christus. Dialog zwischen der Mennonitischen Weltkonferenz und dem LWB, in: KNA-ÖKI Nr. 39, 23.09.2013, Dokumentation: I-XII. 
Das Dokument bietet zunächst eine sehr genau und detaillierte Analyse dessen, was die Aussagen der CA inhaltlich aussagen und gegen welche Positionen sie sich sachlich richten und von wem im 16. Jahrhundert diese Position vertreten worden ist. Dann wird aber ebenso die Wirkungsgeschichte untersucht, und zwar vor allem im Blick auf die politischen Folgen, die ja nicht in der Intention der CA lagen. „Jetzt wurden die Verwerfungen des Augsburger Bekenntnisses, weit davon entfernt, nur theologische Dispute zu definieren, zu Mitteln, um theologische Konformität zu erzwingen und daher Dissidenten zu bestrafen. “30

Auch hier wird - aufbauend vor allem auf dem nationalen Dialog in den USA - deutlich, dass die Verwerfungen in den Artikeln 5, 12 und 17 der CA auf Fehlurteilen über die Täufer des 16. Jahrhunderts beruhen. ${ }^{31}$ Da die nationalen Dialoge zu unterschiedlichen Ergebnissen kommen, was die beiden anderen Artikel betrifft, nämlich CA 9 über die Taufe und CA 16 über das Verhältnis zu Politik und Gesellschaft, geht der Bericht diesen Fragen ausdrücklich nach und tut dies, wie nicht anders möglich, auf der Grundlage der Veränderungen in Kirche, Staat und Gesellschaft, die sich seit dem 16. Jahrhundert ergeben haben und die die Mennoniten ebenso wie die Lutheraner betreffen. Während das Ergebnis für Artikel 16 lautet, dass es zwar weiterhin Unterschiede in der Verhältnisbestimmung zur staatlichen Obrigkeit gibt, die aber keinesfalls unter das Verdikt einer Verwerfung fallen, ${ }^{32}$ wird im Blick auf die Taufe zwar auch die Rede von einer ausdrücklichen Verwerfung vermieden, wohl aber festgehalten, dass die Kirchen hier „noch keinen Weg gefunden [haben], die Kluft zwischen den beiden Kirchen in der Tauflehre und Taufpraxis zu überbrücken“, ${ }^{33}$ so dass hier weiterhin eine kirchentrennende Frage vorliegt. Gerade in diesem Kontext wird deshalb von lutherischer Seite - unbeschadet aller mit der historischen Einordnung wie auch Weiterentwicklung verbundenen Differenzierungen und Klärungen - die unaufgebbare Bindung an die Bekenntnisse und das heißt hier konkret an die inhaltlichen Aussagen der Confessio Augustana als einem Merkmal lutherischer Identität deutlich gemacht. ${ }^{34}$ Entsprechend wird an diese Bindung auch in anderen Dialogen erinnert und damit die Bedeutung gerade des Augsburger Bekenntnisses hervorgehoben. ${ }^{35}$

30 Heilung der Erinnerungen - Versöhnung in Christus, Nr. 104.

31 Vgl. Heilung der Erinnerungen - Versöhnung in Christus, Nr. 130-135 (468-470).

32 Vgl. Heilung der Erinnerungen - Versöhnung in Christus, Nr. 153 (476).

33 Heilung der Erinnerungen - Versöhnung in Christus, Nr. 163 (481).

34 Vgl. Heilung der Erinnerungen - Versöhnung in Christus, Nr. 187 (187).

35 Vgl. etwa: Bericht der von der Lambeth-Konferenz und dem Lutherischen Weltbund autorisierten Gespräche 1970 - 1972 („Pullach-Bericht“), in: DwÜ 1, 54-76, Nr. 29 (58); Adventisten und Lutheraner im Gespräch, Nr. 43 (86); Baptisten und Lutheraner im Gespräch, Nr. 20 (194); Die 


\section{Die Aussagen der Confessio Augustana zu Kirche und Amt}

Es dürfte für niemanden überraschend sein, dass in den verschiedenen ökumenischen Dialogen des Lutherischen Weltbundes gerade die Aussagen der Confessio Augustana zu Kirche und Amt immer wieder zitiert werden, denn sie bilden bekanntlich die Grundlage nicht nur des lutherischen, sondern, wie etwa im Dialog mit den Reformierten und den Anglikanern festgehalten wird, des reformatorischen Kirchenverständnisses insgesamt. ${ }^{36}$

Dabei sind es vor allem drei Sachverhalte, die durchgehend angesprochen werden und in allen Dialogen festgehalten werden.

a) Zum einen wird im internationalen Dialog, wenn es um die Rolle des Amtes geht, von lutherischer Seite der Artikel 7 der Confessio Augustana immer mit den Artikeln 5 und 14 zusammengesehen und die beiden letzteren Artikel - m. E. aus dem historischen Kontext zutreffend - als Aussagen zu ein und demselben Amt verstanden, das damit wesentlich zur Kirche dazugehört. ${ }^{37}$ So heißt es etwa im

Kirche: Gemeinschaft der Gnade, Nr. 19 (236); Alle unter einem Christus. Stellungnahme der Gemeinsamen Römisch-katholischen/Evangelisch-lutherischen Kommission zum Augsburgischen Bekenntnis, 1980, in: DwÜ 1, 323 - 328, Nr. 8 (324); Die Apostolizität der Kirche, Nr. 374 (650). $36 \mathrm{Vgl}$. Bericht der von der Lambeth-Konferenz und dem Lutherischen Weltbund autorisierten Gespräche 1970 - 1972 („Pullach-Bericht“), Nr. 61: „Unsere beiden Kirchen bekräftigen mit nahezu den gleichen Worten (CA VII; 39 Art. XIX), dass die reine Verkündigung des Wortes und die rechte Verwaltung der Sakramente wesentlich und konstitutiv für das Leben der Kirche ist. Wo dies geschieht, sehen wir Kirche.“ (62f.); Zur Gemeinschaft und zum gemeinsamen Zeugnis berufen. Bericht der Gemeinsamen Arbeitsgruppe zwischen dem Lutherischen Weltbund und dem Reformierten Weltbund, 1999-2002, in: DwÜ 3, 111-130, Nr. 28: „Es gibt eine allgemeine Überzeugung, dass eine fundamental-dogmatische Übereinstimmung über die Predigt des Wortes sowie ein gemeinsames Verständnis und eine gemeinsame Praxis der Sakramente ausreichend sei, um Kirchengemeinschaft zu erklären. Wie die Confessio Augustana ausdrücklich hervorhebt, ist grundsätzlich nicht mehr notwendig (satis est). [...] Dieses Grundprinzip sollte jedoch nicht dazu missbraucht werden, die Notwendigkeit zu leugnen, der Einheit der Kirche auch strukturell Ausdruck zu verleihen. Um erfahrbar und anerkannt zu werden, muss die Gemeinschaft sichtbar werden." (119)

37 Alle unter einem Christus, Nr. 16 spricht von ,der Übereinstimmung darin, dass ein besonders, durch Ordination übertragenes Dienstamt für die Kirche konstitutiv ist und nicht zu dem gehört, was das Augsburgische Bekenntnis als ,nicht nötig“ bezeichnet.“(326); Das geistliche Amt in der Kirche. Bericht der Gemeinsamen Römisch-katholischen/Evangelisch-lutherischen Kommission, 1981, in: DwÜ 1, 329-357, Nr. 80: „Das ,satis“ darf nicht so verstanden werden, als sei die Feststellung weiterer Übereinstimmungen etwa nicht mehr legitim. Wenn solche weiteren Übereinstimmungen als ,nicht-notwendig' bezeichnet werden, so soll damit das Wachsen der Einheit in Christus auch in der Gestalt der Kirche nicht verhindert, sondern gerade in rechter Weise frei- 
anglikanisch/lutherischen Dialog: „Die Kirchen der lutherischen Tradition haben folgende Gaben empfangen, in denen sich Gottes Treue ihnen gegenüber konzentriert: die Glaubensbekenntnisse der Alten Kirche, die Bekenntnisschriften des 16. Jahrhunderts und die Kontinuität des ordinierten Amtes, durch welches das Wort Gottes verkündigt und die Sakramente und Riten der Kirche verwaltet worden sind." ${ }^{38}$

Und ebenso wird im lutherisch/katholischen Dokument Kirche und Rechtfertigung festgehalten:

Schon die Confessio Augustana mit ihrem charakteristischen Übergang vom Rechtfertigungsartikel zum Artikel über das kirchliche Amt macht dies klar: Hier wird der rechtfertigende Glaube auf das Evangelium gegründet, welches das kirchliche Amt in Predigt und Sakramenten zu verkündigen hat; und dass „kirchliches Amt“ oder „Predigtamt“ etwas anderes meinen könnte als die kirchliche Institution des ordinierten Amtes - ein Gedanke, der erst im 19. Jahrhundert auftaucht - wird durch CA 14 ausgeschlossen. ${ }^{39}$

b) Zum anderen wird verständlicherweise gerade im Gespräch mit der Anglikanischen Gemeinschaft, mit der katholischen Kirche und sachlich auch mit der Orthodoxie die Frage nach dem Bischofsamt als legitimer Gestalt der Episkopé immer wieder positiv auf CA 28 Bezug genommen, das von den Reformatoren grundsätzlich gewollte Festhalten an der bischöflichen Struktur betont und in-

gegeben werden: als Ausdruck des geistgewirkten Glaubens an das Evangelium, der - wie die Werke des Gerechtfertigten - diesem Glauben folgen sollen. Das so verstandene lutherische ,satis est' steht deshalb dem Verlangen nach der ,Fülle“ kirchlichen Lebens nicht entgegen, sondern schließt den Weg dahin gerade auf.“ (354); Kirche und Rechtfertigung, Nr. 200, Anm. 269: „Apol 7,30 - 37 erläutert CA 7: Worum es in dem, nec necesse est ‘ von CA 7 geht, ist nicht, ob das, was in der Kirche zur Verkündigung des Evangeliums hinzukommt, gut und nützlich für die Kirche ist. Die ,Hauptfrage (Krinomenon)' ist vielmehr, ob es ,not sei zur Seligkeit (necessarius ad iustitiam)'; BSLK 243f.)“ (382); Communion: On Being the Church, Nr. 87: „The ordained ministry rests on Christ's particular commission and, at the same time, stands together with the whole congregation under the Word of God in Christ's service.This ministry is necessary for the church.“ (32); Das Mysterium der Kirche. Wort und Sakramente (Mysterien) im Leben der Kirche. Erklärung der Gemeinsamen Lutherisch/Orthodoxen Kommission, Damaskus/Syrien, 3.-10. November 2000, in: DwÜ 3, 106 - 109, Nr. 9: „In Bezug auf die Manifestation der Kirche in der göttlichen Ökonomie, d.h. in der Heilsgeschichte, bekräftigen wir gemeinsam, dass die Verkündigung des Evangeliums und die Verwaltung der Sakramente durch das ordinationsgebundene Amt in der Kirche zu den wichtigsten Zeichen der Kirche gehören.“ (109); Lutheran-Orthodox Joint Commission, 2017 Common Statement: The Mystery of the Church: F. Ordained Ministry/Priesthood [https:// blogs.helsinki.fi/ristosaarinen/lutheran-orthodox-dialogue/ (16.06.2021)]; Nr. 1; 22; 25.

38 Bericht der Anglikanisch/Lutherischen Konsultation über Episkopé, 1987 („Niagara-Bericht“), in: DwÜ 2, 62-91, Nr. 84 (83).

39 Kirche und Rechtfertigung, Nr. 185 (378). 
sofern die Einordnung in die so genannte historische Sukzession als Möglichkeit für die lutherischen Kirchen akzeptiert. Andererseits aber kann, nicht nur in diesen Dialogen, ${ }^{40}$ sondern auch an anderer Stelle gerade die Offenheit der Aussagen der CA gegenüber den konkreten Strukturen der Kirche betont werden, wie das etwa im baptistisch/lutherischen oder reformiert/lutherischen Dialog der Fall ist. ${ }^{41}$

40 Vgl. neben dem oben genannten „Niagara-Bericht“ auch: Wachsende Gemeinschaft, Nr. 133: „Lutheraner in aller Welt sind zunehmend bereit, die Bedeutung des Episkopats in der apostolischen Sukzession als Zeichen im Dienst der apostolischen Kontinuität und Einheit der Kirche zu würdigen. Die Vereinbarungen zeigen eine wachsende Bereitschaft zur Eingliederung in diese Sukzession; so werden anglikanische und lutherische Bischöfe, die Kirchen mit historischer Bischofssukzession angehören, zur aktiven Teilnahme an Ordinationen oder Einführungen lutherischer Bischöfe in Kirchen eingeladen, die bislang nicht an dieser Sukzession teilhatten. Die Lutheraner sind frei, sich in die historische Bischofssukzession einzugliedern, (1) wenn diese Integration lutherischer Bischöfe in die historische Bischofssukzession nach der gegenseitigen Anerkennung der Kirchen und Ämter und der Erklärung von Kirchengemeinschaft voller Gemeinschaft erfolgt; (2) wenn diese Integration weder ein negatives Urteil über die lutherischen Ämter in der Vergangenheit noch eine Stärkung ihrer kirchlichen Machtposition in der Zukunft impliziert, und (3) wenn es auch weiterhin möglich bleibt, das Amt des Bischofs der Bischöfin und seine ökumenische Bedeutung unterschiedlich auszulegen.“ (164); Die Apostolizität der Kirche, Nr. 269: „Das historische Bischofsamt, das Gegenstand von regionalen ökumenischen Vereinbarungen zwischen Anglikanern und Lutheranern ist, wird von Lutheranern als ein Zeichen für die Apostolizität der Kirche anerkannt. Es wird nicht als eine Garantie für die Apostolizität verstanden, wohl aber als Zeichen, das die ganze Kirche und in ihr besonders die Bischöfe verpflichtet, für diese Apostolizität Sorge zu tragen. So heißt es in der Porvooer Gemeinsamen Feststellung: ,Der Gebrauch des Zeichens der historischen bischöflichen Sukzession allein garantiert nicht die Treue einer Kirche gegenüber jedem Aspekt apostolischen Glaubens, Lebens und Sendung. In der Geschichte der Kirchen, die das Zeichen der historischen Sukzession benutzen, hat es Spaltungen gegeben. Das Zeichen garantiert auch nicht die persönliche Treue des Bischofs. Nichtsdestoweniger bleibt die Beibehaltung des Zeichens eine permanente Aufforderung zu Treue und Einheit, ein Aufruf dazu, die bleibenden Merkmale der Kirche der Apostel zu bezeugen sowie ein Auftrag, sie vollständiger zu verwirklichen. 'So wie ein Bischof zugleich für die Einheit unter den Gemeinden zu seiner Zeit sorgt (synchron) und mit den Ordinationen für die Einheit und Apostolizität der Kirche durch die Zeiten eintritt (diachron), so ist es angemessen, mit dem Zeichen der historischen Sukzession die temporale Dimension der Apostolizität auszudrücken: die Kontinuität der Kirche, die der Heilige Geist wirkt; unter seiner Leitung und seinem Beistand kann der Bischof Diener an der Kontinuität und Apostolizität der Kirche sein.“ (617); The Mystery of the Church: F. Ordained Ministry/Priesthood, Nr. 43; 45.

41 Vgl. etwa: Baptisten und Lutheraner im Gespräch, Nr. 24: „Grundsätzlich schreiben lutherische Bekenntnisschriften (CA 7) keine besondere Struktur der Kirche vor - sei die kongregational, presbyteral oder episkopal -, aber sie bestehen darauf, dass jede Struktur dem Evangelium freien Lauf lassen muss und es nicht behindern darf. So wird angenommen, dass das ordinierte Amt von Gott um des Evangeliums willen gegeben wurde, d.h. um sowohl den freien Lauf als auch die Reinheit des Evangeliums als Wort und Sakrament zu gewährleisten. Deshalb ist die Vollmacht 
c) Und schließlich wird in diesem Zusammenhang ein dritter Aspekt immer wieder erwähnt, nämlich das in CA 7 ausgesprochene bleibende Bestehen der Kirche, ein Aspekt, der zum einen die Verbundenheit der lutherischen Kirchen mit der Kirche der Väter ausdrückt, ${ }^{42}$ der zum anderen aber auch und vor allem theologisch relevant ist für das Verständnis der Indefektibilität der Kirche und ihrer von Gott ermöglichten Treue zum Glauben. ${ }^{43}$ Denn

[f]ür die Reformatoren steht die Frage nach dem Bleiben der Kirche in der Wahrheit im Zusammenhang mit der Gewissheit, „dass alle Zeit müsse eine heilige christliche Kirche sein und bleiben“. Diese Kirche wird verstanden als „Versammlung aller Gläubigen, bei welchen das Evangelium rein gepredigt und die heiligen Sakramente laut dem Evangelium gereicht werden“. Darum ist das Bleiben in der Wahrheit des Evangeliums wesentlich für das Kirchesein der Kirche. ${ }^{44}$

\section{Die Confessio Augustana als Dokument der Einheit}

Dieser Aspekt schließlich ist der Leitgedanke des einzigen Dokuments im internationalen ökumenischen Dialog, das sich ausdrücklich mit der CA befasst, nämlich das Dokument des internationalen lutherisch/katholischen Dialogs Alle

der ordinierten Pfarrer/Pfarrerinnen, das Wort öffentlich zu predigen und zu lehren und die Sakramente zu verwalten, nicht in der Struktur der Kirche, sondern im Evangelium begründet. Die Struktur vermittelt diese Vollmacht, d. h., die Struktur ist immer dem Evangelium untergeordnet.“ (194); Communion: On Being the Church, Nr. 9 (7); Nr. 39 (18f.); The Mystery of the Church: F. Ordained Ministry/Priesthood, Nr. 16.

42 Vgl. Alle unter einem Christus, Nr. 10 (325); Nr. 16 (326); Die Apostolizität der Kirche, Nr. 282: „Lutheraner lehren die Kontinuität der Kirche und betonen, ,dass alle Zeit müsse eine heilige christliche Kirche sein und bleiben'. Deshalb gehört die Geschichte des Amtes von der Zeit des Neuen Testaments an auch zu ihrer Geschichte, die als Geschichte der Kirche ohne den Heiligen Geist nicht zu denken ist.“ (621); Nr. 446 (672f.); Lutheran-Orthodox Joint Commission, The Nature, Attributes and Mission of the Church (15th, Wittenberg 2011), https://blogs.helsinki.fi/ristosaarinen/lutheran-orthodox-dialogue/ (17.06.2021), Nr. 12.

43 Vgl. etwa: Kirche und Rechtfertigung, Nr. 151: „Für Luther ist dieser Glaube an die Unzerstörbarkeit und Permanenz der Kirche als eines heiligen Volkes Gottes eine wesentlich Komponente seines Kirchenverständnisses“ (369); Nr. 174: „Als Geschöpf des stets ,externen“, schöpferischen und von der Treue Gottes getragenen Evangeliums und dessen Verkündigung existiert die Kirche kontinuierlich durch die Zeiten: ,Allezeit [muss] die eine, heilige, christliche Kirche sein und bleiben.“"(375f.).

44 Die Apostolizität der Kirche, Nr. 355 (642); vgl. den ganzen Abschnitt „4.4 Die Kirche, die in der Wahrheit erhalten wird, in der Perspektive der lutherischen Reformation“ (642-655). 
unter einem Christus, ${ }^{45}$ das 1980 aus Anlass der 450-Jahr-Feier der Confessio Augustana veröffentlicht worden ist.

Dieser Text weist gleich zu Beginn zu Recht darauf hin, dass 1530 die Einheit der Kirche „zwar eminent bedroht, aber noch nicht zerbrochen“ war: „Die damaligen ,Religionsparteien` empfanden sich - selbst im Streit und in der Verschiedenheit ihrer Überzeugungen - als ,unter einem Christus' und jener kirchliche Einheit verpflichtet. “46

Dementsprechend nennt das Dokument nicht nur, aufbauend auf den bisherigen lutherisch/katholischen Dialogen, eine Reihe von Gemeinsamkeiten im Verständnis von Rechtfertigung, Herrenmahl, Kirche und Amt, die dann ja weitgehend in den nachfolgenden Dialogen vertieft worden sind, ${ }^{47}$ sondern es hebt gleich zu Beginn den spezifischen Anspruch der Confessio Augustana hervor, nämlich ein Dokument der (synchronen wie diachronen) Einheit und der Verständigung zu sein, ${ }^{48}$ das seine besondere Bedeutung dadurch erhält, dass dieser Text mit dieser Intention zu den verbindlichen Bekenntnissen der lutherischen Kirchen gehört:

Es ist erklärte Absicht des Augsburgischen Bekenntnisses, den Glauben der einen, heiligen, katholischen und apostolischen Kirche zu bezeugen. Es geht nicht um Sonderlehren oder gar um Gründung einer neuen Kirche (CA 7,1), sondern um Reinerhaltung und Erneuerung des christlichen Glaubens - in Einklang mit der Alten Kirche, „auch der römischen Kirche“ und in Übereinstimmung mit dem Zeugnis der Heiligen Schrift. Diese ausdrückliche Intention der Confessio Augustana behält auch für das Verständnis der späteren Lutherischen Bekenntnisschriften Bedeutung. ${ }^{49}$

45 Alle unter einem Christus.

46 Alle unter einem Christus, Nr. 2 (323).

47 Vgl. Alle unter einem Christus, Nr. 14-22 (326f.).

48 Vgl. Alle unter einem Christus, Nr. 7: „Denn dieses Bekenntnis, das Basis und Bezugspunkt der anderen lutherischen Bekenntnisschriften ist, spiegelt wie kein anderes in Inhalt und Struktur den ökumenischen Willen und die katholische Intention der Reformation.“ (324); Vgl. Vom Konflikt zur Gemeinschaft. Gemeinsames lutherisch-katholisches Reformationsgedenken im Jahr 2017. Bericht der Lutherisch/Römisch-katholischen Kommission für die Einheit, Leipzig/Paderborn 2013, Nr. 70: „Das Augsburger Bekenntnis ist ein starkes Zeugnis für die Entschlossenheit der lutherischen Reformatoren, die Einheit der Kirche zu bewahren und innerhalb der einen sichtbaren Kirche zu bleiben. Indem es die Differenz ausdrücklich als von geringerer Bedeutung dargestellt hat, ist dieses Bekenntnis dem ähnlich, was wir heute einen differenzierenden Konsens nennen würden.“ (37).

49 Alle unter einem Christus, Nr. 10 (325); vgl. auch Nr. 8: „Es ist dabei von großem Gewicht, dass dieser ökumenische Wille und die katholische Intention in einem Bekenntnisdokument zum Ausdruck kommen, das auch heute noch - unter und zusammen mit der Heiligen Schrift Lehrgrundlage der lutherischen Kirchen ist und für sie Verbindlichkeit besitzt.“ (324). 
Und man wird hier wohl hinzufügen dürfen, dass die Bedeutung dieses Textes auch darin liegt, dass er nach den altkirchlichen Bekenntnissen der erste Text ist, der am Beginn der lutherischen Bekenntnisschriften des 16. Jahrhunderts steht und er damit gleichsam den Schlüssel für die Lektüre und Interpretation der weiteren Bekenntnisse bildet. ${ }^{50}$

\section{Ausblick}

Ich schließe mit einem kurzen Ausblick, der danach fragt, welche Folgen bzw. theologischen Herausforderungen sich aus diesem Blick auf die Rolle der CA im ökumenischen Dialog ergeben.

Zum einen erscheint es als eine weiterhin offene Frage, und damit sage ich nichts Neues, eine genauere Verhältnisbestimmung der Confessio Augustana und der anderen lutherischen Bekenntnisse zur Theologie Martin Luthers zu geben. Mit dem Jahr 2017 im Rücken und auf das Jahr 2030 zugehend stellt sich hier eine Aufgabe, die auch der ökumenische Dialog meiner Wahrnehmung nach bisher kaum angesprochen hat. Auf der einen Seite wird die Verbindung zwischen beiden selbstverständlich und zur Recht bejaht, wie es etwa das Dokument Martin Luther - Zeuge Jesu Christi ausspricht: „Die Einsicht, dass die Confessio Augustana ,eine Übereinstimmung in zentralen Glaubenswahrheiten' zwischen Katholiken und Lutheranern widerspiegelt (Papst Johannes Paul II., 1980; Exekutivkomitee des Lutherischen Weltbundes, 1981), hilft, auch wesentliche Einsichten Luthers gemeinsam zu bejahen. ${ }^{\text {"51 }}$ Auf der anderen Seite muss der Status der CA als ausdrückliches und verbindliches Bekenntnis der lutherischen Kirchen unterschieden werden von der Geltung der Aussagen Luthers, auch und gerade, wenn man die einheitsstiftende Intention der CA wie auch ihre Bedeutung im Rahmen des gesamten Konkordienbuchs ernstnimmt.

50 Vgl. Burkhard Neumann, Die Bedeutung der „Confessio Augustana“ und der „Apologie“ Melanchthons für die Ökumene. Historische Beobachtungen und Überlegungen aus der Sicht eines katholischen Systematikers, in: Günter Frank/Stephan Meier-Oeser (Hg.), Konfrontation und Dialog. Philipp Melanchthons Beitrag zu einer ökumenischen Hermeneutik, Leipzig 2006 (Schriften der Europäischen Melanchthonakademie 1), 125-138, 129.

51 Martin Luther - Zeuge Jesu Christi. Wort der Gemeinsamen Römisch-katholischen/Evangelisch-lutherischen Kommission anlässlich des 500. Geburtstages Martin Luthers, 1983, in: DwÜ 2, 444-451, Nr. 5 (445); vgl. auch: Heilung der Erinnerungen - Versöhnung in Christus, Nr. 187: „Lutheraner sind auch heute sehr dankbar für die Lehre des Evangeliums, die sie von Martin Luther empfangen haben, und sie sehen sich weiterhin seinem Verständnis des Wortes Gottes verpflichtet, wie es vor allem in ihrer Verpflichtung auf das Augsburger Bekenntnis und die anderen Bekenntnisschriften zum Ausdruck kommt.“ (491). 
Zum anderen stellt m.E. gerade der lutherisch/mennonitische Dialog ein Beispiel dafür dar, was eine historisch wie theologisch gründliche Auslegung des Augsburger Bekenntnisses einschließlich eines durchaus kritischen Blicks auf ihre Wirkungsgeschichte beitragen kann zu einem wirklichen Verständnis der in der Confesio Augustana gemachten und weiterhin als verbindlich geltenden Aussagen. Was in diesem Text geschieht, lässt sich vergleichen mit der Intensität, mit der das Projekt Lehrverurteilungen - kirchentrennend zu Beginn der 80-Jahre des vergangenen Jahrhunderts die Lehrverurteilungen des 16. Jahrhunderts untersucht und im Blick auf den aktuellen Stand der Lehre in den Kirchen bewertet hat. ${ }^{52}$ Unter diesem Niveau sollte bzw. dürfte ein theologisch verantworteter Blick auf die CA heute nicht mehr möglich sein. Es wäre darum umso wichtiger, ihn in ökumenischer Gemeinschaft anzugehen.

52 Karl Lehmann/Wolfhart Pannenberg (Hg.), Lehrverurteilungen - kirchentrennend?, Bd. 1: Rechtfertigung, Sakramente und Amt im Zeitalter der Reformation und heute, Freiburg i.B./ Göttingen ${ }^{3} 1988$ (Dialog der Kirchen 4); Karl Lehmann, (Hg.), Lehrverurteilungen - kirchentrennend?, Bd. 2: Materialien zu den Lehrverurteilungen und zur Theologie der Rechtfertigung, Freiburg i.B./Göttingen 1989 (Dialog der Kirchen 5); Wolfhart Pannenberg (Hg.), Lehrverurteilungen - kirchentrennend?, Bd. 3: Materialien zur Lehre von den Sakramenten und vom kirchlichen Amt, Freiburg i.B./Göttingen 1990 (Dialog der Kirchen 6); Wolfhart Pannenberg/Theodor Schneider (Hg.), Lehrverurteilungen - kirchentrennend?, Bd. 4: Antworten auf kirchliche Stellungnahmen, Freiburg i.B./Göttingen 1994 (Dialog der Kirchen 8). 
